\title{
Interruptive alerts: only one part of the solution for clinical decision support
}

\author{
Yogini H Jani, ${ }^{1,2}$ Bryony Dean Franklin (D) 1,3
}

${ }^{1}$ Research Department of Practice and Policy, University College London School of Pharmacy, London, UK ${ }^{2}$ Centre for Medicines Optimisation Research and Education, University College London Hospitals NHS Foundation Trust, London, UK ${ }^{3}$ Centre for Medication Safety and Service Quality, Imperial College Healthcare NHS Trust/ UCL School of Pharmacy, London, UK

\section{Correspondence to} Dr Yogini H Jani, Research Department of Practice and Policy, University College London School of Pharmacy, London WC1N 1AX, UK; y.jani@ucl.ac.uk

Accepted 2 August 2021 Published Online First 12 August 2021

\section{Sinked}

- http://dx.doi.org/10.1136/ bmjqs-2020-012283

Check for updates

(c) Author(s) (or their employer(s)) 2021. No commercial re-use. See rights and permissions. Published by BMJ.

To cite: Jani YH, Franklin BD. BMJ Qual Saf

2021;30:933-936.
It is widely accepted that electronic health records, computerised provider order entry (CPOE) and electronic-prescribing systems represent a way forward for patient safety. Even relatively basic electronic-prescribing systems have been shown to improve patient safety by eliminating the 'technical' prescription writing errors of clarity and completion, such as those related to poor handwriting or missing information. ${ }^{12}$ However, reductions in errors relating to clinical decisions are likely to require more advanced systems incorporating clinical decision support systems (CDSS), which may also be linked to patient-specific information. A recent overview highlights that CDSS '....is comprised of software designed to be a direct aid to clinical-decision making, in which the characteristics of an individual patient are matched to a computerised clinical knowledge base and patientspecific assessments or recommendations are then presented to the clinician for a decision'. ${ }^{3}$ Within electronic-prescribing and CPOE systems, such recommendations are often presented to the user as interruptive pop-up alerts, including those relating to allergies, drug-drug interactions and potentially inappropriate doses.

The systematic review by Cerqueira and colleagues ${ }^{4}$ in this issue of $B M J$ Quality \& Safety focuses on the effectiveness of such interruptive alerts on prescribing behaviour in outpatient and ambulatory care settings. The review specifically excluded other types of CDSS as well as non-interruptive alerts. The authors identified only nine comparative studies of interruptive alerts that met their inclusion criteria, of which three were randomised controlled trials, with randomisation at either the level of the practice or the prescriber. Eight of the nine studies were from North America. The effectiveness of interruptive alerts on prescriber behaviour was conceptualised as prescriber acceptance of the alerts leading to changes in prescribing practices, medication costs and/or prescribing errors. The authors conclude that seven of the nine studies demonstrated significant provider behaviour change, although effect sizes were often small, and confidence intervals wide. However, despite the review's broad conceptualisation of effectiveness, none of the studies assessed patients' health outcomes. The authors of the review also note that in the few studies that captured prescriber feedback, the alerts were often considered inappropriate, intrusive and of limited value, with only one study reported to include more positive feedback. Cerqueira and colleagues do not discuss prescriber feedback in depth, but one interpretation of these findings could be that interruptive CDSS alerts change prescriber behaviour with little evidence for patient benefit and at the expense of prescriber irritation.

As highlighted by Cerqueira and colleagues, ${ }^{4}$ interruptive alerts are associated with a number of problems in both inpatient and outpatient settings. As well as affecting prescriber experience, these include increasing the time required for prescribing tasks, high rates of over-rides (some of which may be inappropriate, putting patients at risk), low alert sensitivity and alert fatigue. ${ }^{5}$ Other unintended negative consequences, such as delays in prescribing and adverse outcomes due to inappropriate acceptance of irrelevant alerts, have also been reported. ${ }^{6}$ In the hospital setting, greater benefits have been reported for more tailored alerts, such as drug allergy alerts or dosing recommendations based on patient characteristics, 
with higher acceptance rates suggesting increased alert specificity. ${ }^{7}$

Given these issues, the general principle of interruptive alerts as a way of improving safety warrants further exploration. Distractions and interruptions are contributory factors to errors and reduce situational awareness in clinical workflow. ${ }^{8}$ Yet, interruptive alerts continue to be used in electronic prescribing and CPOE systems to present clinical decision support with the aim of improving safety. This apparent paradox may be due to a fundamental distinction between what we generally think of as 'interruptions', which detract attention away from the primary task, ${ }^{9}$ and 'interruptive alerts', which provide an intentional disruption as part of the primary task. In the former, the primary task often needs to be suspended for a period of time, whereas the pause is more disruptive with interruptive CDSS alerts, to intentionally prompt a review of the primary task. In the context of integrated electronic health records, where users may be accessing the systems for various reasons and therefore be presented with alerts unrelated to their primary task, the distinction of whether alerts act as interruptions or disruptions becomes more important and requires further research.

Most research in this field has evaluated the impact of single CDSS features such as alerts or decision-guiding recommendations in isolation, rather than a composite of different types of CDSS, or in the context of wider system design with very few replication studies. ${ }^{10} 11$ We therefore suggest that the widespread use of electronic health record systems and emerging evidence for alternative CDSS designs, ${ }^{12} 13$ combined with advances in the fields of clinical informatics and artificial intelligence (AI) make this the right time to recalibrate our conceptualisation of CDSS. This recalibration involves four key considerations. First, we need to move away from the widely held association of CDSS with interruptive pop-up alerts, and instead focus on broader design concepts, including any on-screen tools that support and facilitate clinical decision-making or improve adherence to recommended processes of care. Such facilitative decision support design can include a range and mix of proactive and reactive approaches (table 1). This may include making best use of more basic CDSS features such as drug dictionaries, formularies and preference lists, through the application of nudge theory and behaviour change frameworks to nudge users towards safe and appropriate prescribing. Second, better use should be made of more advanced features, such as patientdependent or user-dependent rule-based prepopulated doses, order sets and tiered or graded alerts with optimised alert sensitivity and specificity; these may deliver greatest gain with minimum disruption. ${ }^{14}$ Third, current evidence on the challenges, disadvantages, limitations and usability flaws of the different types of CPOE and CDSS should be used to improve system design. This should include

Table 1 Terminology and approaches used in clinical decision support system (CDSS) design

\begin{tabular}{|c|c|c|}
\hline Design feature & Description & Example \\
\hline Interruptive alert & $\begin{array}{l}\text { Pop-up; temporarily stops the primary task; requires } \\
\text { acknowledgement or cancellation to continue. }\end{array}$ & Drug allergy or drug interaction alerts. \\
\hline Facilitative interruptive alert & $\begin{array}{l}\text { As above but facilitates next steps by providing options } \\
\text { for follow-on actions, for example, alternative choices. }\end{array}$ & $\begin{array}{l}\text { As above but suggests suitable alternative, for example, } \\
\text { second-line antibiotic choice for patients with penicillin } \\
\text { allergy. }\end{array}$ \\
\hline Non-interruptive alert & $\begin{array}{l}\text { Alert that appears on screen but does not interrupt or } \\
\text { stop the primary task. }\end{array}$ & $\begin{array}{l}\text { Sidebar presenting additional information that may be } \\
\text { actioned after the primary task is complete. }\end{array}$ \\
\hline Graded or tiered alerts & $\begin{array}{l}\text { Alerts (either interruptive or non-interruptive) that } \\
\text { include information about the likelihood or severity of } \\
\text { the risk associated with the information being presented. }\end{array}$ & $\begin{array}{l}\text { Contraindications graded as higher risk than } \\
\text { precautionary warnings. }\end{array}$ \\
\hline Drug dictionary & List of all the medicines available for prescribing. & $\begin{array}{l}\text { Commercial and non-commercial drug databases that } \\
\text { facilitate choice of medicines to prescribe. }\end{array}$ \\
\hline Formulary & $\begin{array}{l}\text { List of all the medicines available for prescribing in that } \\
\text { organisation or for that user or patient group. }\end{array}$ & $\begin{array}{l}\text { Organisation-level formulary that restricts the choice of } \\
\text { medicines available to prescribe. }\end{array}$ \\
\hline Preference list & $\begin{array}{l}\text { Selection of medicines that are commonly or frequently } \\
\text { prescribed by the user or user group. }\end{array}$ & $\begin{array}{l}\text { Preference list for acute admissions presenting with } \\
\text { myocardial infarction, set at a system level, to nudge the } \\
\text { user to prescribe by protocol. }\end{array}$ \\
\hline Prepopulated doses or frequencies & $\begin{array}{l}\text { Recommended dose or frequency is suggested when a } \\
\text { medicine is selected; may be based on age or indication. }\end{array}$ & $\begin{array}{l}\text { Nudge to prescribe the appropriate low molecular } \\
\text { weight heparin dose for prophylaxis. }\end{array}$ \\
\hline Order sets & $\begin{array}{l}\text { Structured and standardised collection of orders for a } \\
\text { given condition; usually based on evidence or guidelines. }\end{array}$ & $\begin{array}{l}\text { Postoperative medication orders for specific procedures } \\
\text { to nudge the user to prescribe by protocol. }\end{array}$ \\
\hline Forcing function & $\begin{array}{l}\text { Requires action to progress, that is, restricts and prevents } \\
\text { further actions until the issue has been addressed; often } \\
\text { used in combination with interruptive or non-interruptive } \\
\text { alerts. }\end{array}$ & $\begin{array}{l}\text { Mandatory entry of essential information, for example, } \\
\text { entry of age for a paediatric prescription. }\end{array}$ \\
\hline
\end{tabular}


using human factor approaches to learn from studies of unanticipated behaviours to explore how the prescriber was led to such behaviours and to improve system design to support those behaviours that lead to positive outcomes. Finally, the application of AI and machine learning tools may also provide more refined, adaptive decision support systems that can iteratively enhance decision-making recommendations based on patient and prescriber characteristics. This could support alert-based CDSS with greater sensitivity and specificity, or to optimise non-alertbased CDSS such as prepopulated doses and order sets.

Given some of the unique features of CDSS, the design of evaluation studies also requires careful consideration. Evaluations need to consider broader CDSS design features including the timing and context of the information provided to influence prescribers, ${ }^{15}$ rather than studying the impact of individual features, such as alerts, in isolation. ${ }^{16}$ Comparative studies of nudge frameworks with interruptive alerts or forcing functions are also needed to assess the potential pitfalls of each approach. For example, there may also be unintended consequences with nudge interventions, if users accept default or suggested doses without adjusting doses when needed for specific patients. Randomised controlled trials that consider an intervention containing different elements as one overall 'treatment' are also unlikely to be useful to study the unique features of CDSS in real-world settings, where it may be challenging to control for cross-over effects due to patient care being delivered by different teams and different individual prescribers. Although modelling approaches have been recommended, these too are limited in the assessment of both actual prescriber behaviours and other factors such as cognitive workload. ${ }^{17} 18$ Future research is needed using mixed methods and adaptive study designs such as platform trials ${ }^{19} 20$ to assess the impact of these interventions on patient outcomes, used in combination with rapid ethnography to understand the behavioural aspects. These alternative research approaches allow the continuous study of multiple interventions or components and in effect, the development of a learning health system $^{21}$ that embeds knowledge generation into routine practice.

In conclusion, we argue that interruptive alerts are only one part of the solution to improve patient safety through electronic-prescribing and CPOE systems. Further safety improvements can only be achieved if CDSS design is facilitative, with minimal cognitive disruption or burden, and includes greater consideration of human factor principles. There is also the need for better research, measuring actual impact on patient outcomes.
Correction notice The paper has been corrected since it was published online first. The funding statement has been updated.

Twitter Bryony Dean Franklin@BryonyDF

Funding BDF is supported by the National Institute for Health Research (NIHR) Imperial Patient Safety Translational Research Centre and the NIHR Health Protection Research Unit in Healthcare Associated Infections and Antimicrobial Resistance at Imperial College in partnership with Public Health England (PHE).

Disclaimer The views expressed are those of the author(s) and not necessarily those of the NHS, the NIHR, PHE or the Department of Health and Social Care.

Competing interests None declared.

Patient consent for publication Not required.

Provenance and peer review Commissioned; internally peer reviewed.

ORCID iD

Bryony Dean Franklin http://orcid.org/0000-0002-2892-1245

\section{REFERENCES}

1 Jani YH, Ghaleb MA, Marks SD, et al. Electronic prescribing reduced prescribing errors in a pediatric renal outpatient clinic. J Pediatr 2008;152:214-8.

2 Franklin BD, O'Grady K, Donyai P, et al. The impact of a closed-loop electronic prescribing and administration system on prescribing errors, administration errors and staff time: a before-and-after study. Qual Saf Health Care 2007;16:279-84.

3 Sutton RT, Pincock D, Baumgart DC, et al. An overview of clinical decision support systems: benefits, risks, and strategies for success. NPJ Digit Med 2020;3:17.

4 Cerqueira O, Gill M, Swar B, et al. The effectiveness of interruptive prescribing alerts in ambulatory CPOE to change prescriber behaviour \& improve safety. BMJ Qual Saf 2021;30:1038-46.

5 van der Sijs H, Aarts J, Vulto A, et al. Overriding of drug safety alerts in computerized physician order entry. J Am Med Inform Assoc 2006;13:138-47.

6 Powers EM, Shiffman RN, Melnick ER, et al. Efficacy and unintended consequences of hard-stop alerts in electronic health record systems: a systematic review. J Am Med Inform Assoc 2018;25:1556-66.

7 Carli D, Fahrni G, Bonnabry P, et al. Quality of decision support in computerized provider order entry: systematic literature review. JMIR Med Inform 2018;6:e3.

8 Kellogg KM, Puthumana JS, Fong A, et al. Understanding the types and effects of clinical interruptions and Distractions recorded in a multihospital patient safety reporting system. J Patient Saf 2018. doi:10.1097/PTS.0000000000000513. [Epub ahead of print: 07 Jul 2018].

9 Altmann EM, Trafton JG. Memory for goals: an activationbased model. Cogn Sci 2002;26:39-83.

10 Marcilly R, Ammenwerth E, Vasseur F, et al. Usability flaws of medication-related alerting functions: a systematic qualitative review. J Biomed Inform 2015;55:260-71.

11 Coiera E, Tong HL. Replication studies in the clinical decision support literature-frequency, fidelity, and impact. J Am Med Inform Assoc 2021. doi:10.1093/jamia/ocab049. [Epub ahead of print: 06 Jul 2021].

12 Miller K, Mosby D, Capan M, et al. Interface, information, interaction: a narrative review of design and functional 


\section{Editorial}

requirements for clinical decision support. J Am Med Inform Assoc 2018;25:585-92.

13 Kubben P, Dumontier M, Dekker A. Fundamentals of clinical data science, 2019.

14 Hussain MI, Reynolds TL, Zheng K. Medication safety alert fatigue may be reduced via interaction design and clinical role tailoring: a systematic review. J Am Med Inform Assoc 2019;26:1141-9.

15 Lamprell K, Tran Y, Arnolda G, et al. Nudging clinicians: a systematic scoping review of the literature. J Eval Clin Pract 2021;27:175-92.

16 Marcilly R, Ammenwerth E, Roehrer E, et al. Evidence-based usability design principles for medication alerting systems. BMC Med Inform Decis Mak 2018;18:69.

17 Medlock S, Wyatt JC, Patel VL, et al. Modeling information flows in clinical decision support: key insights for enhancing system effectiveness. J Am Med Inform Assoc 2016;23:1001-6.

18 Ancker JS, Edwards A, Nosal S, et al. Effects of workload, work complexity, and repeated alerts on alert fatigue in a clinical decision support system. BMC Med Inform Decis Mak 2017;17:36.

19 Adaptive Platform Trials Coalition. Adaptive platform trials: definition, design, conduct and reporting considerations. Nat Rev Drug Discov 2019;18:797-807.

20 Park JJH, Harari O, Dron L, et al. An overview of platform trials with a checklist for clinical readers. J Clin Epidemiol 2020;125:1-8.

21 (US), I.o.M. Digital infrastructure for the learning health system: the foundation for continuous improvement in health and health care: workshop series summary, 2011. 